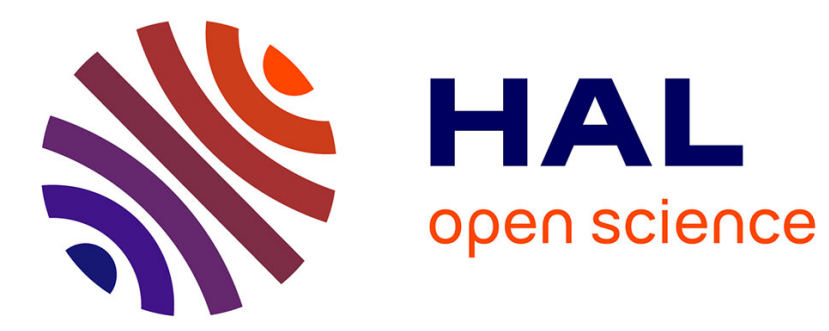

\title{
La recevabilité d'une expertise scientifique aux Etats-Unis
}

\author{
Rafael Encinas de Munagorri
}

\section{To cite this version:}

Rafael Encinas de Munagorri. La recevabilité d'une expertise scientifique aux Etats-Unis. Revue internationale de droit comparé, 1999, pp.621-632. hal-01329198

\section{HAL Id: hal-01329198 \\ https://hal.science/hal-01329198}

Submitted on 29 Nov 2017

HAL is a multi-disciplinary open access archive for the deposit and dissemination of scientific research documents, whether they are published or not. The documents may come from teaching and research institutions in France or abroad, or from public or private research centers.
L'archive ouverte pluridisciplinaire HAL, est destinée au dépôt et à la diffusion de documents scientifiques de niveau recherche, publiés ou non, émanant des établissements d'enseignement et de recherche français ou étrangers, des laboratoires publics ou privés. 


\title{
La recevabilité d'une expertise scientifique aux États-Unis
}

\author{
Article paru in Revue internationale de droit comparé, 1999, pp. 621-632.
}

\begin{abstract}
Résumé
Comment garantir la fiabilité d'une expertise scientifique présentée devant les tribunaux ? Au terme d'une évolution de plus d'un siècle, la Cour suprême des États-Unis a posé des conditions de recevabilité qui conduisent les juges à apprécier la validité des connaissances scientifiques de l'expert.
\end{abstract}

How to make sure that scientific expertise is reliable in the courtroom trial ? After a more than century evolution, the United States Supreme Court ruled a standard of admissibility for scientific evidence that leads judges to assess the validity of expert's scientific knowledge.

1. Le fait et le droit sont comme les extrémités d'un arc que seuls les juristes savent manier. Que l'un des éléments leur échappe et la flèche de la justice s'égare dans la nature. Si le domaine du droit reste chasse gardée, la science a envahi celui des faits. Nos gestes de tous les jours s'accomplissent au rythme des objets issus des sciences appliquées : conduire une voiture, avaler un médicament, composer un numéro de téléphone. Que la science et ses techniques puissent dévoiler la vérité des faits est alors un juste retour des choses.

2. Les juges sont censés connaître le droit, mais non les faits du litige. Et lorsque ces derniers sont complexes, le recours à un expert est nécessaire, voire indispensable². Toutes les branches du droit sont concernées par l'expertise : du droit commercial au droit de l'environnement, du droit pénal au droit des brevets. Et que dire du droit de la filiation sur la base duquel a pu être ordonné une exhumation à des fins probatoires $^{3}$ ? En matière de responsabilité civile la décision semble parfois aussi reposer tout entière sur la démonstration scientifique d'un lien de causalité. Si la victime parvient à établir par voie d'expertise que tel produit ou substance a été la cause du dommage subi, elle aura toutes les chances d'obtenir réparation. Il convient néanmoins de rappeler que la décision revient aux juges et non à l'expert dont l'avis n'est jamais, en droit, déterminant. Reste que si les juges entendent

1. Ce texte a pour origine une communication présentée à la Cour de cassation le 12 juin 1998 lors du colloque "l'expertise entre science et droit" organisé à l'initiative du Groupement de recherches (GDR) "Sciences et Droit". Sa mise à jour a été rendue possible par une subvention du Ministère de l'éducation nationale et de la recherche, DSPT 7. Je tiens à remercier Christine Noiville et Gilles Lhuilier pour leur lectures attentatives et la qualité de leurs observations.

2. Rappelons que les tribunaux ne sont pas l'unique instance de décision où s'exerce l'expertise scientifique. voir sur ce point Marie-Angèle HermitTe, "L'expertise scientifique à finalité politique. Réflexion sur l'organisation et la responsabilité des experts", Justice, 1997, n8, p.79; et en langue anglaise l'ouvrage de Sheila JASANOFF, The Fifth Branch. Science advisers as Policymakers, 1990, Harvard University Press, 1994.

3. C. A. Paris, 6 nov. 1997, D. 1998, p. 122, note Philippe MaLAurie. 
conserver leurs pouvoirs d'interpréter et de qualifier la réalité, ils sont attirés par l'aptitude de l'expert à établir un fait sur des bases scientifiques. Le recours à l'expertise peut toutefois aboutir à une impasse lorsque rien ne peut être affirmé où lorsque les experts ne sont pas unanimes. Les juges sont alors confrontés à un problème redoutable : comment décider en situation d'incertitude ? Pris en étau entre l'obligation de trancher le litige sous peine de déni de justice et celle de se fonder sur des éléments de fait aussi exacts que possible, ils sont placés dans l'embarras. En définitive, comme on a pu souvent le faire remarquer, les rapports entre les juges et les experts ne vont pas de soi.

3. Civilisation à haute teneur scientifique et juridique, les États-Unis sont à l'avantgarde des problèmes liés à l'usage judiciaire de la connaissance scientifique. Le tournant décisif de ces dernières années est l'arrêt Daubert ${ }^{4}$ rendu le 28 juin 1993 par lequel la Cour Suprême des États-Unis a bouleversé les conditions de recevabilité d'une expertise scientifique ${ }^{5}$. Le sens du revirement est clair : alors que les juges pouvaient s'en remettre à ce qui est généralement admis par les spécialistes en un domaine donné, ils sont désormais invités à s'assurer que les experts appelés devant les tribunaux présentent les garanties scientifiques suffisantes.

4. La solution prend place au sein de règles de procédure qui s'appliquent aux juridictions fédérales, mais dont l'influence est forte sur les juridictions des États membres ${ }^{6}$. Le système retenu est celui de liberté de la preuve. Sauf exceptions prévues par la Constitution des États-Unis, le Congrès ou la Cour suprême ellemême, toute preuve pertinente est recevable. De plus, les parties peuvent prouver par voie de témoignage. La remarque est d'importance car elle concerne aussi l'expertise. A la différence du droit français c'est en principe sous la forme procédurale d'un témoignage que l'expert se fait entendre devant la cour et les jurés. L'expert n'est certes pas un témoin ordinaire qui est censé connaître les faits

4. Daubert v. Merrell Dow Pharmaceuticals, Inc. 113 S. Ct. 2786 (1993). Dans les notes qui vont suivre, seules les décisions de justices seront citées selon le système de référence en vigueur aux États-Unis. Toutes les traductions -sans nul doute perfectibles- sont de notre main et apparaissent entre guillemets.

5. Voir Kenneth R. Foster et Peter W. Huber, Judging Science : Scientific Knowledge and the Federal Courts, M.I.T. Press 1997 ; tous les ouvrages relatifs aux rapports de la science et du droit consacrent désormais des développements à la solution, voir Steven Goldberg, Culture Clash. Law and Science in America, New York University Press, 1994, pp. 20-23 ; Sheila JASAnOfF, Science at the Bar : Law, Science, and Technology in America, Harvard University Press, 1995, pp. 57-67 auxquels il faut ajouter ceux de scientifiques ou de médecins, Angel MArcia, Science on Trial. The Clash of Medical Evidence and the Law in the Breast Implant Case, New York, Norton, 1996, pp. 125-132.

6. Dénommé Federal Rules of Evidence, (FRE), ce code est une compilation et une refonte des règles de procédures applicables devant les juridictions fédérales des États-Unis. Il est en vigueur depuis 1975. 
de l'espèce pour les avoir vus ou entendus, mais son avis n'en prend pas moins la forme d'un témoignage destiné à éclairer les juges.

5. Ces précisions prennent tout leur relief dans une procédure à caractère accusatoire où les parties ont l'initiative de réunir les faits à l'appui de leurs prétentions ${ }^{\text {. }}$. Chaque partie produit ses expertises, apporte ses preuves, démontre sa vérité. C'est ainsi que le débat peut prendre la tournure d'une bataille d'experts choisis par les parties puisqu'il peut être fait librement recours à des experts en qualité de témoin. Encore faut-il préciser que la compétence scientifique des témoins présentés en qualité d"experts" peut être inégale : du spécialiste mondialement reconnu au charlatan de circonstance. L'accroissement de ces derniers a d'ailleurs conduit à dénoncer l'intrusion d'une science de pacotille devant les prétoires. Elle est d'autant plus difficile à combattre que tous les experts bénéficient, en qualité de témoin, d'une immunité qui les met à l'abri d'une action en responsabilité. Déterminer les conditions de recevabilité d'une expertise devient alors crucial, surtout lorsque l'expert est à même d'influencer les jurés, dont on rappellera le rôle prépondérant aux États-Unis dans la détermination des faits ${ }^{10}$.

6. Les questions sont alors nombreuses et trouvent écho dans notre propre système juridique. Quelles sont les garanties que doit présenter un expert, et quels moyens les juges disposent-ils pour s'assurer de la fiabilité de l'expertise ? Quels sont les contours et le domaine de l'expertise scientifique? Est-elle une expertise particulière à laquelle s'applique un régime spécial ou constitue-t-elle le droit commun de l'expertise ? De manière plus générale, on peut se demander comment les juges parviennent à concilier la recherche de la vérité des faits avec l'incertitude générée par l'évolution des connaissances. Au-delà de problèmes relatifs au droit de la preuve, il s'agit aussi de prendre en compte les nouvelles articulations entre la science et le droit, la connaissance et la décision, le savoir et le pouvoir. On ne fera ici que signaler le mouvement du droit positif amorcé aux États-Unis. Il consiste à rendre plus restrictives les conditions par lesquelles une expertise

\footnotetext{
7. Règle $n^{\circ} 702 \mathrm{du}$ FDE : "If scientific technical, or other specialized knowledge will assist the trier of fact to understand the evidence or to determine a fact in issue, a witness qualified as and expert by knowledge, skill, experience, training, or education may testify thereto in the form of an opinion or otherwise." Observons dès à présent que le texte distingue la connaissance scientifique, de celle technique ou spécialisée. La remarque est d'importance et l'un des enjeux actuels consiste à savoir si les règles applicables à l'expertise scientifique sont valables pour toutes les expertises.

8. Voir en langue anglaise l'excellent ouvrage de Geoffrey C. HaZARd et Michele TARUffo, American Civil Procedure : An Introduction, Yale University Press, 1993, p. 86 et s. ; et en langue française, Droit des États-Unis, sous la direction d'Alain Levasseur, Paris, Dalloz, 2 éd., 1994. éd. nº 236.

9. Aux États-Unis, le fléau est connu sous celui de la Junk science. Pour une dénonciation virulente du phénomène, Peter W. Huber, Galileo's Revenge : Junk Science in the Courtroom, 1991, New York, Basic Books, 1993. En français, on pourrait parler de science camelote, science dépotoir, on encore de science pacotille.

10. Voir sur ce point les ouvrages cités supra note 8, resp. p.17, 71 et $n^{\circ} 243$.
} 
scientifique est recevable devant les tribunaux. L'évolution sera présentée en trois périodes successives.

\section{I - La période pragmatique (jusqu'en 1923) : l'expertise scientifique est recevable si elle est délivrée par un expert compétent}

7. Dès les premières expertises, les juges ont été confrontés à deux difficultés : s'assurer de la compétence de l'expert, éviter les controverses sans fin entre spécialistes.

8. Lorsque toute personne peut se prétendre "expert" sans être inscrite sur une liste officielle $^{11}$, rien ne garanti sa compétence. Aussi les juges ont bien vite posé les garanties que doit présenter l'expert pour que son avis soit recevable. Ils ont pour cela retenu une solution fort simple : sera considéré comme expert celui qui gagne sa vie par l'exercice de ses compétences. Ce critère, qui a pu être formulé comme le commercial marketplace test, repose sur un raisonnement élémentaire en accord avec une société liant étroitement les compétences et la réussite professionnelle. Si une personne exerce une activité ou une profession qui suppose un tel savoir, si elle gagne de l'argent ou acquiert de la notoriété sur le marché par l'exercice de ses compétences, c'est le signe qu'elle dispose, en une matière donnée, d'un savoir qui est supérieur à la moyenne, car sans cela on ne ferait pas appel à ses services. La réussite professionnelle laisse présumer que la personne appelée à témoigner est à même d'éclairer la cour et les jurés.

Autant dire que tout professionnel pouvait témoigner au profit d'une partie en qualité d'expert. Le recours à l'expertise connut alors une expansion rapide. Ce qui n'allait pas manquer de créer des inconvénients.

9. Dès 1858 les juges de la Cour suprême déplorent la versatilité mercantile des experts et la perte de temps à les écouter afin d'évaluer leurs mérites ${ }^{12}$. La finalité de l'expertise ne doit pas être perdue de vue : il s'agit pour les juges de se forger une opinion sur les faits de l'espèce. Et dès lors qu'ils s'estiment assez informés, l'expert a joué son rôle. Les juges ne sauraient être contraints à l'écouter davantage ${ }^{13}$.

11. Aux États-Unis, et malgré des projets recurrents, la désignation d'un expert inscrit sur une liste officielle n'a jamais eu les faveurs des juges, ni surtout des parties. Ces dernières souhaitent en effet conserver l'initiative du procès dont elles bénéficient en vertu du caractère accusatoire de la procédure. Aujourd'hui, il ne serait fait appel à des experts "appointés" que dans seulement $20 \%$ des cas. voir Reference Manual of Scientific Expertise, éd. Federal Judicial Center, 1994, p. 535.

12. Winans v. New York \& Erie Railroad, 62, U.S. 88 (1858), p. 101 :"Experience has shown that opposite opinions of persons professing to be experts may be obtained to any amount; and it often occurs that not only many days, but even weeks, are consumed in cross-examination, to test the skill or knowledge of such witnesses and the correctness ot their opinions, wasting the time and wearying the patience of both court and jury and perplexing instead of elucidating, the questions involved in the issue".

13. Ibid: "A judge may obtain information from them -the experts-, if he desire it, on matters which de does not clearly comprehend, but cannot be compelled to receive their opinions as matter of evidence" Signalons 
Les conséquences de cette position peuvent être illustrées par un procès criminel où un expert prétendait apporter, au titre de la défense, la preuve de la folie mentale de l'accusé. Il fut alors demandé à l'expert d'exposer l'état de la science médicale enseignée sur ce point. Le principe d'une telle question fut rejeté par les juges : l'expert n'a pas à répondre de l'état de la science médicale en général, son seul témoignage suffit. La Cour Suprême confirma la décision : "Dès lors qu'un témoin s'est présenté lui-même comme expert et étant donné son avis professionnel ...il serait excessif de l'interroger sur ce que d'autres hommes de science ont dit en pareille matière, ou au regard de ce qui est enseigné en général sur ce point, ou encore de permettre à des livres de science d'être offerts comme moyens de preuve" ${ }^{114}$.

10. A partir du moment où l'expert était réputé compétent, son expertise devenait en principe recevable et elle pouvait être prise en compte à des fins probatoires. Autrement dit, les juges pouvaient se contenter d'une opinion isolée. Ils évitaient certes par là d'entrer dans les arcanes du débat scientifique. Mais les conditions de recevabilité d'une expertise demeuraient par trop accueillantes ; ce qui laissait la porte ouverte à des décisions fondées sur la base d'expertises fantaisistes. Les conditions de recevabilité d'une expertise ne pouvaient que connaître des restrictions.

\section{La période positive (1923-1993) : l'expertise scientifique est recevable si elle repose sur des connaissances généralement admises}

11. A partir de 1923 et pendant près de soixante dix ans, le droit s'ordonne autour d'un critère plus exigeant : le savoir de l'expert doit correspondre aux connaissances généralement admises par la communauté de spécialistes.

12. La solution retenue à partir de l'arrêt Frye ${ }^{15}$ est la suivante : non seulement l'expert doit connaître les opinions d'autres experts, mais son témoignage n'est recevable que s'il exprime la position de la communauté des spécialistes à laquelle il appartient. Le critère du general acceptance standard se substitua à celui du commercial marketplace test.

que si les experts ne peuvent forcer les juges à les écouter, l'inverse n'est plus vrai. L'un des débats actuels aux États-Unis consiste à savoir dans quelle mesure les juges peuvent enjoindre les scientifiques, le cas échéant contre leur gré, à révèler des données pour les besoins de la justice, voir sur ce point le numéro spécial de Law \& Contemporary Problems, 1996, vol. 59, nº 3.

14. Davis v. United States, 165 U.S. 373 (1896), p. 377: "After a witness has once qualified himself as an expert and given his own professional opinion in reference to which he has seen or heard or upon hypothetical questions, then it is ordinarily opening the door to too wide an inquiry to interrogate him as to what other scientific men have said upon same matters, or in respect to the general teachings of science thereon, or to permit books of science to be offered in evidence".

15. Frye v. United States, 293 F. 1013 (D.C. Cir. 1923). Que l'arrêt n'ait pas été rendu par la Cour suprême des États-Unis, mais par la cour fédérale d'un État membre n'a pas fait obstacle à ce que la solution retenue fasse jurisprudence. 
L'exposé des faits ayant donné lieu à l'arrêt Frye suffit à convaincre des mérites de l'évolution du droit en ce sens. Pour établir son innocence dans une affaire où il était accusé de meurtre, Frye eut recours au témoignage d'un expert. Ce dernier prétendait établir à l'aide d'un détecteur de mensonge que Frye n'avait pas menti. Tout le problème était donc de savoir si la technique utilisée- il s'agissait en l'espèce d'un instrument permettant de mesurer la tension artérielle - pouvait servir de moyen de preuve. Le maintien de la position antérieure aurait pu conduire les juges à déclarer recevable une telle expertise. La Cour fédérale de Columbia refusa de l'admettre par une formulation dont les commentateurs ont souvent souligné la maladresse : "the thing from whitch the deduction is made must be sufficiently established to have gained general acceptance in the particular field in which it belongs". En l'espèce, le détecteur de mensonge ne relevait pas des connaissances généralement admises par les scientifiques, du moins pas assez pour servir de moyen de preuve. L'expertise fut donc déclarée irrecevable.

13. Les juridictions fédérales adoptèrent assez vite ce nouveau critère. A partir de 1923 l'expertise scientifique ne devint recevable que si elle correspondait aux données acquises de la science, ou plus exactement au savoir généralement partagé, à ce qui est tenu pour vrai au sein d'une communauté de spécialistes.

Comme souvent en matière juridique, une solution nouvelle s'impose par son aptitude à concilier deux impératifs contradictoires. Elle permit ici de garantir une fiabilité à l'expertise sans pour autant contraindre les juges à se plonger dans les méandres de la science. Les juges pouvaient ainsi écarter des prétoires les imposteurs et charlatans assez doués pour vendre leur "science camelote", sans pour autant qu'ils n'aient à se prononcer par eux-mêmes sur la valeur d'une expertise.

14. La simplicité du critère est seulement apparente. Et les difficultés apparurent bien vite au cours de sa mise en oeuvre. C'est que le nouveau critère déplace la problème plus qu'il ne le résout. Car si les juges doivent apprécier la conformité des connaissances scientifiques invoquées par l'expert avec celles généralement admises, comment pourraient-ils le faire sans s'informer de l'état de la science au moment du litige ? En période de stabilité des connaissances scientifiques, on peut certes penser que le critère reste applicable. Mais qu'en est il lorsque les connaissances se diversifient et évoluent à une cadence soutenue ?

$\mathrm{Du}$ fait de l'évolution des connaissances, les juges furent menacés d'un double écueil dans l'application du critère de general acceptance standard. Soit l'application était trop restrictive et conduisait à déclarer l'expertise irrecevable. C'est l'archaïsme qui l'emporte et la justice se prive des dernières avancées de la recherche. Soit l'application était trop souple et conduisait à déclarer l'expertise recevable. C'est l'illusion du progrès qui l'emporte et la justice se prive de savoirs plus fiables et mieux reconnus. 
15. Il faut enfin se demander s'il est bien opportun en matière preuve de calquer les conditions de recevabilité d'une expertise sur l'état des connaissances scientifiques à un moment donné. On évite certes par là toute distorsion entre la science utilisée devant les tribunaux et celle en cours dans les laboratoires : la preuve juridique est en quelque sorte repliée sur la preuve scientifique. Les juges peuvent se fonder sur l'état positif des connaissances. Mais nombreux sont les auteurs à penser que cette superposition est illusoire et que les finalités probatoires ne sont pas les mêmes pour la science et pour le droit ${ }^{16}$. Sans compter que les scientifiques impliqués dans la recherche ne sont pas ceux qui jouent le rôle d'expert devant les tribunaux. De plus, quand bien même ce serait le cas, le contexte même du procès où ils sont appelés à intervenir les entraînent parfois à modifier leur attitude : la blouse de laboratoire est alors troquée pour la robe d'avocat ${ }^{17}$.

16. A partir de 1975, date à laquelle entre en vigueur la codification des règles de preuve applicables devant les juridictions fédérales, la jurisprudence issue de l'arrêt Frye perdit de son autorité. Il faut dire que le critère de "connaissances généralement admises" ne fut pas retenu dans le Code des règles fédérales et l'incertitude s'instaura un moment : le silence du législateur voulait-il dire que le critère de general acceptance était abandonné ? Et si oui, quelles étaient alors les nouvelles conditions de recevabilité d'une expertise scientifique ? A ces deux interrogations la Cour suprême des États-Unis a apporté une réponse tranchée.

\section{III - La période critique (à partir de 1993) : l'expertise scientifique est recevable si elle est fondée sur des connaissances valides}

17. L'arrêt Daubert ${ }^{18}$ est porteur d'un revirement spectaculaire qui bouleverse, au delà de l'expertise scientifique devant les juridictions fédérales, l'ensemble de la pratique de l'expertise judiciaire aux États-Unis. La motivation de la Cour suprême est exemplaire à cet égard. Après avoir confirmé la mise à l'écart du critère de "connaissances généralement admises", elle définit les nouvelles conditions de recevabilité d'une expertise scientifique ${ }^{19}$. Les juges sont désormais invités à un double examen : vérifier que l'expertise repose sur des connaissances scientifiques,

\footnotetext{
16. L'affirmation est récurente. Voir par ex. dans l'arrêt Daubert, op. cit. p. 2798. "there are important differences between the quest for truth at the courtroom and the quest for truth in the laboratory. Scientific conclusions are subject to perpetual revisions. Law, on the other hand, must resolve disputes finally and quickly." Parmi de nombreuses contributions on signalera la synthèse de l'un des pionners de l'étude des rapports science et droit aux États-Unis, Lee Loevinger, "Standard of Proof in Science and Law", 32 Jurimetrics Journal, 1992, p. 323.

17. Sur les changements d'attitude des scientifiques induits par leur présence en qualité d'experts au sein d'une procédure accusatoire, Dan L. BuRK, "When Scientists Act Like Lawyers : the Problem of Adversary Science", 33 Jurimetrics Journal, 1993, p. 363.

18. Daubert, op. cit., note $\mathrm{n}^{\circ} 4$.

19. Sur le plan des sources du droit, la Cour suprême ne fait qu'interpréter la règle $n^{\circ} 702$ reproduite supra note 7 qui pose les cas où un témoin peut être admis en qualité d'expert.
} 
s'assurer que l'expertise permettra de comprendre et de déterminer les faits de la cause $^{20}$.

18. Dans l'affaire Daubert, du nom de la victime, la question était de savoir si un médicament destiné à éviter les nausées pendant la grossesse (la Bendictine fabriquée par les laboratoires Merrell Dow Pharmaceuticals) était à l'origine de malformations à la naissance. Procès en responsabilité classique où la réponse dépendait de la preuve du rapport de cause à effet entre le médicament et le dommage subi. A l'appui de sa prétention la victime produisit huit expertises. Toutes n'avaient pas été publiées dans des revues à caractère scientifique et certaines avaient même été réalisées ad hoc, pour les seules fins du litige. La compagnie pharmaceutique, quant à elle, présenta de nombreuses études ayant donné lieu à publication ${ }^{21}$. Déboutée en première instance et en appel, la victime saisit avec succès la Cour suprême des États-Unis : l'expertise dont elle se prévalait ne pouvait être déclarée irrecevable. L'affaire fut donc à nouveau jugée sur le fond sans du reste donner satisfaction à la victime .

19. Au-delà de la solution de l'espèce, c'est la motivation de la Cour qui retiendra notre attention ${ }^{22}$. En vertu de la règle $\mathrm{n}^{\circ} 702 \mathrm{du}$ Code fédéral ${ }^{23}$, l'expert ne peut être admis à témoigner que si la connaissance dont il se prévaut est scientifique. Or, selon la Cour suprême, ces deux mots ont un sens juridique. Les juges sont ainsi désormais invités à apprécier si les deux éléments sont bien réunis. Pour cela, la Cour suprême entend préciser le sens des termes en question ${ }^{24}$. Afin de mettre en oeuvre la règle de preuve, les juges fédéraux doivent désormais exercer "une évaluation préliminaire pour déterminer si le raisonnement ou la méthodologie qui sert de base au témoignage est scientifiquement valide et peut être appliquée aux faits de la cause" ${ }^{25}$. Ce qui suppose que puisse être demandé in limine litis un examen de la recevabilité de l'expertise présentée par l'une des parties ${ }^{26}$. L'exigence d'une appréciation par les juges du caractère "valide" de la science sur laquelle se

20. Daubert, op. cit., p. 2795." Faced with a proffer or expert scientific testimony, then, the trial judge must determine ...whether the expert is proposing to testify to (1) scientific knowledge that (2) will assist the trier of fact to understand or determine a fact in issue".

21. Ajoutons que le médicament était assez répandu, (17 millions de femmes l'auraient utilisé entre 1958 et 1983) de même que la malformation (1 cas sur 1000 naissances environ ).

22. Pour un commentaire détaillé de la décision voir Bert BlACK, Francisco J. Ayala and Carol SAFFran-BrinKs, "Science and the Law in the Wake of Daubert: A New Search for Scientific Knowledge", 72 Texas Law Review, 715, (1994). et de manière plus synthétique, Lee LOEVINGER, "Science as Evidence", 35 Jurimetrics Journal , 1995, p.153 . voir du point des nouvelles obligations pesant sur les juges, David L. FaIGman, "Mapping the Labyrinth of Scientific Evidence", Hastings Law Journal, 1995, p. 555.

23. voir supra note 7.

24. Daubert, op.cit., p. 2795 :" the adjective "scientific" implies a grounding in the methods and procedures of science. Similarly, the word "knowledge" connotes more than subjective belief or unsupported speculation". 25. Ibid., p. 2796. The rule 702 "entails a preliminary assessment of whether the reasoning or methodology underlying the testimony is scientifically valid and of whether that reasoning or methodology properly can be applied to the facts in issue". 
fonde l'expert est singulière. Elle signifie que les juges sont désormais gardiens non seulement de la pertinence de l'expertise scientifique au regard des faits de l'espèce, mais aussi de sa fiabilitée ${ }^{27}$. Comme la Cour le précise, lorsqu'il est question "de preuve scientifique dans un litige, la «fiabilité de la preuve» sera fondée sur sa «validité scientifique ${ }^{\prime 28}$.

20. Afin de conforter les juges dans leur nouvelle mission, la Cour suprême entend se montrer rassurante : Nous avons confiance dans les aptitudes des juges fédéraux pour réaliser cette appréciation. Sous la forme d'observations générales elle prend néanmoins le soin de fournir les lignes directrices qui doivent prévaloir à cet examen. La méthode proposée est celle du faisceau d'indices. Aucun critère n'est déterminant, mais tous concourent à apprécier si l'expertise proposée à une validité scientifique.

Le premier indice est de savoir "si la théorie ou technique -utilisée par l'expertpeut être (et a été) testée." Par une citation à Sir Karl Popper, la Cour suprême adopte la conception prônée par le philosophe des sciences : le critère du statut scientifique d'une théorie est sa réfutabilité, c'est-à-dire son aptitude à se soumettre à une expérience scientifique susceptible d'un résultat positif ou négatif. Le second indice est de savoir si "la théorie ou technique à donné lieu à une évaluation par les pairs et à publication"29. La Cour ajoute que "la publication n'est pas une condition sine qua non de la recevabilité." Mais le simple fait d'avoir été soumis à la critique par le jeu des évaluations internes à la communauté scientifique est un gage de la fiabilité de l'expertise.

Le troisième indice s'ajoute aux deux premiers lorsque les techniques scientifiques utilisées par les experts se prêtent à une appréciation statistique. Les juges doivent alors "considérer le pourcentage d'erreur connu ou potentiel de techniques scientifiques particulières" ${ }^{130}$.

\footnotetext{
26. L'audition préalable de l'expert par les juges hors de la présence des jurés offre l'avantage d'écourter la procédure lorsque l'expertise est déclarée irrecevable. Dans ce cas, les jurés ne sont mêmes pas convoqués. En ce sens, Mark S. KleIN, "The Revolution in Practice and Procedure : «Dauberts Hearings»", in Shepard's Expert \& Scientific Evidence, p. 655.

27. Daubert, p. 2795 :"the trial judge must ensure any and all scientific testimony or evidence admitted is not only relevant, but reliable".

28. Ibid., p. 2795. note 9 :"In a case involving scientific evidence, «evidentiary reliability» will be based upon «scientific validity»".

29. Observons que si la publication n'est pas une garantie absolue de fiabilité, la réciproque est aussi vraie en ce sens que la fiabilité scientifique ne suppose pas l'existence d'une publication. Il est des théories trop spécifiques, trop récentes, ou encore d'un intérêt trop limité pour avoir donné lieu à publication, et qui ne reposent pas moins sur des bases scientifiques solides. Aucun obstacle juridique ne s'oppose à ce que des expertises soient fondées sur des théories non publiées. Ce qui n'exclut pas que les scientifiques puissent aider les juges dans leurs évaluations, voir Lawrence S. PINSKY, "The Use of Scientific Peer Review and Colloquia to Assist Audges in the Admissibility Gatekeeping Mandated by Daubert", Houston Law Review, vol. 34, n², 1997, p. 527.

30. A titre d'illustration, la Cour suprême mentionne la prise en compte du pourcentage d'erreur d'une reconnaissance de la voix par spectrographie.
} 
La quatrième indice correspond à l'ancien critère de "general acceptance" qui survit, mais comme un indice parmi d'autres. Ce qui est généralement admis dans une communauté scientifique laisse seulement présumer que le connaissance est valide sur le plan scientifique. Il va sans dire que la présomption est alors simple, et non irréfragable.

21. Par un mouvement progressif et sans doute irréversible, quelques années ont suffit pour que soient adoptées par les juridictions fédérales les conditions de recevabilité posées dans l'arrêt Daubert ${ }^{31}$. Il est sans doute trop tôt pour mesurer l'ensemble des conséquences de la nouvelle jurisprudence confirmée par l'arrêt Joiner $^{32}$. Trois enseignements peuvent néanmoins être d'ores et déjà tirés.

En premier lieu, les exigences posées dans le cadre de l'expertise scientifique au sens strict ont pu être étendues ailleurs. C'est ainsi que des expertises proposées par des médecins, des ingénieurs, des économistes, des historiens, des psychanalystes ou encore des graphologues se sont vu appliquer les nouvelles conditions de recevabilités ${ }^{3}$. Dans une société ou la science fournit le modèle dominant de la connaissance, l'évolution était pour le moins prévisible. Qu'elle se réclame ou non de "scientifique", l'expertise se fonde sur une connaissance. Et de nos jours la connaissance est intimement liée à la rigueur de la science et de ses méthodes ${ }^{34}$.

En second lieu, la crainte que les juges ne puissent assumer leur nouvelle mission est sans doute excessive ${ }^{35}$. Car il n'est pas demandé aux juges d'avoir une connaissance approfondie des contenus de la science. Il suffit qu'ils aient assez de repères pour évaluer si les méthodes utilisées à l'occasion d'une expertise sont fiables d'un point de vue scientifique et appropriées au regard des faits de l'espèce. Des efforts de formation ont été entrepris en ce sens comme en témoigne la réalisation et la diffusion d'un manuel de référence destiné à aider les juges à évaluer la fiabilité d'une expertise scientifique présentée comme moyen de

31. Voir par ex. Heather G. Hamilton, "The Movement from Frye to Daubert : "Where Do the States Stand ?", 38 Jurimetrics Journal, 1998, p. 201.

32. General Electric Co v. Joiner, 118 S. Ct, 512 (1997). voir en particulier p. 517 où les juges rappelent dans un premier temps l'obligation posée dans l'arrêt Daubert supra note 27, avant d'en préciser la portée : "while Fereral Rules of Evidence allow districts courts to admit a somewhat broader range of scientific testimony that would have been admissible under Frye, they leave in place the "gatekeeper» role of the trial judge in screening such evidence".

33. La jurisprudence n'est pas uniforme sur ce point. Et la question reste posée de savoir s'il y a lieu de distinguer les sciences dures des sciences douces. Pour un tableau contrasté des décisions sur ce point, voir Stephen A. Saltzburg et al., Federal Rules of Evidence Manual, vol. 2, 17 éd., 1998, p. 1241.

34. Cela est d'ailleurs si vrai qu'il est difficile de concevoir, dans le cadre de l'expertise judiciaire, un fait qui soit autrement prouvé que sur des bases scientifiques. Au lieu de prendre en compte des faits scientifiquement établis, on imagine mal aujourd'hui que les juges puissent retenir des faits émotionellement ressentis ou théologiquement révèlés.

35. L'affirmation n'est pas partagée par tous, y compris au sein de la Cour suprême. Voir les opinions dissidentes formulées par le juge Stevens dans les arrêts Daubert, op.cit., p. 2800 et Joiner, op.cit., p. 521. 
preuve $^{36}$. De plus les juges conservent une latitude assez importante dans l'application de la jurisprudence Daubert. Et les plus réticents semble être parvenus à conserver leurs habitudes. Reste que le tournant est pris. Et le temps parviendra sans doute à modifier les comportements afin que les juges s'approprient les connaissances nécessaires pour apprécier les mérites d'une expertise scientifique. Enfin, et de manière plus générale, la jurisprudence Daubert modifie l'attitude des juges à l'égard de la science. La justice a pour objectif de résoudre des litiges particuliers et le juge doit parfois trancher dans l'incertitude des faits, c'est-à-dire sans connaître les résultats encore controversés de l'activité scientifique ${ }^{37}$. Il prend alors, au deux sens du terme, le risque de devancer le mouvement des connaissances généralement admises et d'accueillir une expertise valide et fiable ; soit bientôt confirmée par l'évolution des sciences et de ses méthodes, soit bientôt démentie par le même mouvement. Cela est sans doute moins insolite qu'il n'y paraît de prime abord : la vérité des faits scientifiques n'est elle pas elle-même, et par définition, provisoire et sans cesse remise en cause?

22. En conclusion, il est possible de présenter l'évolution des conditions de recevabilité d'une expertise scientifique aux États-Unis par l'attitude des juges à l'égard de la science. Au cours d'une première période qualifiée de pragmatique, alors que les sciences appliquées restent discrètes dans la vie quotidienne, les juges ne font qu'apprécier la compétence de l'expert sur le plan professionnel. Attitude d'indifférence à l'égard de la science dont ils peuvent se permettre de tout ignorer, ou de connaître seulement par une vue d'ensemble. Au cours d'une seconde période où la science prospère sous la bannière d'un progrès devenu accessible et visible sur le plan matériel, les juges doivent apprécier si les connaissances de l'expert correspondent à celle généralement admises par la communauté de spécialistes. Attitude de confiance où ils doivent s'informer de l'état positif des connaissances scientifiques. Au cours de la période la plus récente, où la science et les technologies sont omniprésentes, les juges doivent apprécier la validité des connaissances scientifiques utilisées par l'expert. Attitude critique qui suppose de disposer d'une culture scientifique élémentaire. En définitive, au cours de ces trois périodes définies à gros traits, les conditions de recevabilité se cumulent plus

36. Reference Manual on Scientific Evidence, Federal Judicial Center, éd. 1994.

Conçu comme un document de formation professionnel établi pour aider les juges lorsqu'ils sont confrontés à des expertises scientifiques, ce volumineux manuel de 637 pages a été publié peu de temps après l'arrêt Daubert. Il présente d'une part le cadre général de la procédure relative aux règles de preuve, et expose d'autre part des domaines d'expertise particuliers : épidémiologie, toxicologie, analyse d'ADN, inférences statistitiques, étude par enquêtes, analyse à régression multiple, estimation du préjudice économique. Pour un compte rendu de ce manuel voir le numéro spécial de la revue Jurimetrics Journal, vol. 36, 1996, p. 121.

37. La Cour suprême apporte dans l'arrêt Daubert une précision importante : "it would be unreasonable to conclude that the subject of scientific testimony must be known to a certainty; arguably there are no certainties in science". Citant des amicus curiae issus de groupements scientifiques tels que l'American Association for the Advancement of Science, ou la National Academy of Sciences, la Cour dissocie à juste raison le caractère scientifique d'une expertise de la certitude de ses résultats. 
qu'elles ne se substituent les unes aux autres. Pour conserver leurs prérogatives sur les faits, les juges semblent devoir s'investir dans une compréhension de plus en plus approfondie de la science ${ }^{38}$. Au-delà de l'évolution, il importe de bien prendre en compte l'élan mutuel par lequel la compréhension entre juristes et scientifiques est appelée à s'établir. Est-il besoin de rappeler, par delà leurs traditions et utopies respectives, que le droit et la science participent de l'unité de la culture ${ }^{39}$ ?

Rafael Encinas de Munagorri Professeur à l'université de Bourgogne

38. En ce sens Sheila JASANOFF, "What Judges Should Know about Sociology of Science", Jurimetrics Journal, 1992, p. 345.

39. Déclaration des îles sous le vent, par Gilles Lhuilier et moi-même, C.U.P.F., 1998. 